\title{
Observational diagnostics of elongated planet-induced vortices with realistic planet formation time-scales
}

\author{
Michael Hammer ${ }^{\oplus},{ }^{1 \star}$ Paola Pinilla, ${ }^{1} \dagger$ Kaitlin M. Kratter $^{1}$ and Min-Kai Lin ${ }^{\oplus 2}$ \\ ${ }^{1}$ Steward Observatory, University of Arizona, Tucson, AZ 85721, USA \\ ${ }^{2}$ Institute of Astronomy and Astrophysics, Academia Sinica, Taipei 10617, Taiwan
}

Accepted 2018 October 29. Received 2018 October 26; in original form 2018 March 24

\begin{abstract}
Gap-opening planets can generate dust-trapping vortices that may explain some of the latest discoveries of high-contrast crescent-shaped dust asymmetries in transition discs. While planet-induced vortices were previously thought to have concentrated shapes, recent computational work has shown that these features naturally become much more elongated in the gas when simulations account for the relatively long time-scale over which planets accrete their mass. In this work, we conduct two-fluid hydrodynamical simulations of vortices induced by slowly growing Jupiter-mass planets in discs with very low viscosity $\left(\alpha=3 \times 10^{-5}\right)$. We simulate the dust dynamics for four particle sizes spanning $0.3 \mathrm{~mm}$ to $1 \mathrm{~cm}$ in order to produce synthetic ALMA images. In our simulations, we find that an elongated vortex still traps dust, but not directly at its centre. With a flatter pressure bump and disruptions from the planet's overlapping spiral density waves, the dust instead circulates around the vortex. This motion (1) typically carries the peak off-centre, (2) spreads the dust out over a wider azimuthal extent $\geq 180^{\circ}$, (3) skews the azimuthal profile towards the front of the vortex, and (4) can also create double peaks in newly formed vortices. In particular, we expect that the most defining observational signature, a peak offset of more than $30^{\circ}$, should be detectable $>30$ per cent of the time in observations with a beam diameter of at most the planet's separation from its star.
\end{abstract}

Key words: hydrodynamics - methods: numerical - protoplanetary discs.

\section{INTRODUCTION}

In the last few years, a combination of individual observations and surveys using the Atacama Large Millimeter Array (ALMA) have imaged a large sample of transition discs, many of which have largescale asymmetries in the dust at $\mathrm{mm}$ and sub-mm wavelengths (e.g. Casassus et al. 2013; van der Marel et al. 2013; Pérez et al. 2014; Ansdell et al. 2016; Barenfeld et al. 2016; Pascucci et al. 2016). One possible explanation for these features is that they are dusttrapping vortices induced by giant planets that triggered the Rossby Wave instability (RWI: Lovelace et al. 1999; Li et al. 2000, 2001) as they opened up gaps in low-viscosity discs (e.g. Li et al. 2005; de Val-Borro et al. 2007).

The idea that these asymmetries are signatures of planets can be tested in multiple ways. Observational studies have attempted to validate these features as vortices by showing that the dust in these asymmetries becomes more concentrated at larger grain sizes (van der Marel et al. 2015). Meanwhile, computational studies have also tried to support this explanation by showing that synthetic images

\footnotetext{
^E-mail: mhammer44444@gmail.com
}

$\dagger$ Hubble Fellow. generated from simulations of a vortex's dust distribution closely match the images taken by ALMA (Bae, Zhu \& Hartmann 2016). None the less, even if an asymmetry is a vortex, it may not have been created by a planet. The build-up of gas at a dead-zone boundary can also generate a vortex (e.g. Lyra \& Mac Low 2012; Regály et al. 2012; Miranda et al. 2017). Furthermore, alternate ideas such as the presence of a close-in companion star adding eccentricity to the disc (Ataiee et al. 2013; Ragusa et al. 2017; Price et al. 2018) are also possible. These scenarios that do not rely on planets may still prove to be better explanations for some, if not all of these asymmetries.

To understand if disc asymmetries can be evidence for undetected planets, it is useful to simulate the dynamics of dust in a disc in order to create synthetic images at mm wavelengths - the range of emission that traces particle sizes expected to be trapped by vortices in the outer part of a disc. Yet, previous efforts to simulate images of these vortices have neglected the time it takes for a planet to grow to Jupiter-size, a key parameter in determining the properties of a vortex (Hammer, Kratter \& Lin 2017). Computational models of core accretion - the preferred method for forming most gas giants - show that a giant planet will obtain most of its mass during its runaway growth phase, which can last for thousands of orbits or longer in discs with the low viscosities needed for vortices to form 
(Pollack et al. 1996; Lissauer et al. 2009). Taking these time-scales into account, Hammer et al. (2017) found that a giant planet can first trigger the RWI while it is still in its growth phase. At this point, it will create a weaker, elongated vortex compared to the stronger, more concentrated vortices induced by planets grown in less than 100 orbits. With these differences in the shape of the gas vortex, Hammer et al. (2017) expect the dust component seen in observations to appear differently as well.

In this work, we investigate the impact of a gas vortex having an elongated structure on dust trapping. We generate synthetic images of both elongated vortices and concentrated vortices in order to identify unique observational signatures that can be used to differentiate these two cases.

The paper is organized as follows. In Section 2, we describe our two-fluid (gas and dust) 2D hydrodynamical simulations and our method for generating synthetic images. In Section 3, we compare the two classes of vortices in two-fluid simulations and synthetic dust continuum images. We propose several criteria needed to classify an observed asymmetry as an elongated vortex. In Section 4, we apply our results to existing and future observations. In Section 5, we summarize our results.

\section{METHODS}

Following a similar set-up to Hammer et al. (2017), we use the twofluid version of the hydrodynamical code (Masset 2000; Zhu et al. 2012) to simulate vortex formation induced by rapidly and slowly accreting planets (see Section 2.1). We then produce synthetic images of our simulated asymmetric dust discs that can be compared to existing and future observations (see Section 2.2).

\subsection{Two-fluid hydrodynamics}

\subsubsection{Numerical methods}

FARGO uses the finite difference method and adaptive time-steps to solve the Navier-Stokes equations in 2D cylindrical polar coordinates $(r, \phi)$. The code takes advantage of the FARGO algorithm to speed-up simulations by subtracting out the average azimuthal velocity when calculating the Courant-Friedrich-Levy condition that is used to determine the largest allowed time-step.

This variation of FARGO (Zhu et al. 2012) builds upon the original code by adding a dust component as a secondary pressureless fluid. In addition to the source terms felt by the gas, the dust also feels radial and azimuthal drag forces due to its coupling with the gas. These terms are given by

$\frac{\partial v_{\mathrm{r}, \mathrm{d}}}{\partial t}=-\frac{v_{\mathrm{r}, \mathrm{d}}-v_{\mathrm{r}, \mathrm{g}}}{t_{\mathrm{s}}}$,

$\frac{\partial v_{\theta, \mathrm{d}}}{\partial t}=-\frac{v_{\theta, \mathrm{d}}-v_{\theta, \mathrm{g}}}{t_{\mathrm{s}}}$,

where $v_{\mathrm{r}}$ and $v_{\theta}$ are the radial and azimuthal velocity components and $v_{\mathrm{d}}$ and $v_{\mathrm{g}}$ are the dust and gas components, respectively. Since we only simulate small particles, the stopping time always falls in the Epstein regime (Weidenschilling 1977) and is given in the mid-plane by

$t_{\mathrm{S}}=\frac{\mathrm{St}}{\Omega}=\left(\frac{\pi}{2} \frac{\rho_{\mathrm{d}} s}{\Sigma_{\mathrm{g}}}\right) \frac{1}{\Omega}$,

where $\rho_{\mathrm{d}}$ is the physical density of each dust grain (which we take to be $1 \mathrm{~g} \mathrm{~cm}^{-3}$ ), $s$ is the size of each grain, $\Sigma_{\mathrm{g}}$ is the local surface density of the gas, and $\Omega$ is the local angular velocity. The Stokes number $\mathrm{St}$ is the dimensionless form of the stopping time. Besides being affected by drift, the dust can also diffuse through the disc due to turbulence. This effect acts as an extra source term (Clarke \& Pringle 1988) for the evolution of the density and is given by

$\frac{\partial \Sigma_{\mathrm{d}}}{\partial t}=\nabla \cdot\left(D \Sigma_{\mathrm{g}} \nabla\left(\frac{\Sigma_{\mathrm{d}}}{\Sigma_{\mathrm{g}}}\right)\right)$

where the diffusion coefficient, $D$, can be approximated as the turbulent viscosity, $v$, for small particles with $\mathrm{St} \ll 1$ (Youdin \& Lithwick 2007). The evolution of the gas is not affected by the dust since we do not include dust feedback. ${ }^{1}$ We address the limitations of neglecting feedback in Sections 3.2 and 4.1. For more details on the implementation of the dust component, see section 2.1.2 of Zhu et al. (2012).

\subsubsection{Gas component}

Each simulation begins with a locally isothermal gas disc having a radial density profile of $\Sigma_{\mathrm{g}}(r)=\Sigma_{\mathrm{g}, 0}\left(r / r_{\mathrm{p}}\right)^{-1}$, where $\Sigma_{0}$ is the initial surface density at the orbital radius of the planet, $r_{\mathrm{p}}$. The value of $\Sigma_{\mathrm{g}, 0}$ is set by the total disc mass in the domain, which we choose to be $M_{\mathrm{d}}=0.002=2 M_{\mathrm{p}}$. We set the disc's aspect ratio to a constant value $h \equiv H / r=0.06$, which empirically maximizes the strength of the vortex for fixed planet and disc parameters (Fu et al. 2014). A constant aspect ratio corresponds to a temperature profile that scales as $T(r) \propto r^{-1}$. The viscosity is set to $v=10^{-7}$ in dimensionless units of $r_{\mathrm{p}}^{2} \Omega_{\mathrm{p}}$. This value corresponds to $\alpha=3 \times 10^{-5}$ near the location of the planet for the standard alpha prescription of $v=$ $\alpha H^{2} \Omega_{\mathrm{p}}$ (Shakura \& Sunyaev 1973). Our previous work found that this viscosity permitted slowly growing planets to induce long-lived elongated vortices for the parameters used in this study (Hammer et al. 2017).

We simulate an annulus of a disc across a domain that spans $r$ $\in[0.2,5.7] r_{\mathrm{p}}$ in radius and $\phi=[0,2 \pi]$ in azimuth. The annulus is resolved by $N_{\mathrm{r}} \times N_{\phi}=2048 \times 3072$ arithmetically spaced grid cells in the radial and azimuthal directions, respectively, which is comparable to the grid sizes used in recent studies of dust evolution in vortices (e.g. Barge et al. 2017; Miranda et al. 2017; Surville \& Mayer 2018). This is high enough to resolve both the disc's scale height $H(r)$ and the planet's Hill radius by at least 22 cells in the vicinity of both the planet and the vortex. Additionally, the strong dependence of the vortex properties on the viscosity in Hammer et al. (2017) verifies that the resolution is sufficient for the prescribed viscosity to dominate over any numerical viscosity from the code.

\subsubsection{Dust component}

Alongside the gas disc, we initialize a dust fluid component that follows the same power-law density profile over the same domain, except with a lower surface density of $\Sigma_{\mathrm{d}, 0}=\Sigma_{\mathrm{g}, 0} / 100$. We do not prescribe a viscosity to the dust fluid, leaving it to diffuse only through the gas turbulence as described in Section 2.1.1. In a given simulation, the dust fluid represents particles of a single fixed size specified as an input parameter (see Section 2.1.6).

\footnotetext{
${ }^{1}$ However, the gas simulations are still not identical for each dust size
} because numerical noise propagates over time. 


\subsubsection{Planet}

At a radius of $r_{\mathrm{p}}$, we place a Jupiter-mass planet $\left(M_{\mathrm{p}}=M_{\mathrm{J}}\right)$ on a fixed circular orbit around a solar mass star $\left(M_{*}=\mathrm{M}_{\odot}\right)$ with an orbital angular velocity of $\Omega_{\mathrm{p}}=\sqrt{\mathrm{GM}_{\odot} / r_{\mathrm{p}}^{3}}$, where $G$ is the gravitational constant. This is equivalent to an orbital period of $T_{\mathrm{p}} \equiv 2 \pi / \Omega_{\mathrm{p}}$. For simplicity, we set $r_{\mathrm{p}}=\Omega_{\mathrm{p}}=G=M_{*}=1$ when used in the code. The planet's gravitational potential is given by

$\phi_{\mathrm{p}}(\mathbf{r})=\frac{\mathrm{G} M_{\mathrm{p}}}{\sqrt{\left(\mathbf{r}-\mathbf{r}_{\mathbf{p}}\right)^{2}+r_{\mathrm{s}}^{2}}}$,

where $r_{\mathrm{s}}=0.6 h$ is the smoothing length.

We prescribe the planet's mass, $m_{\mathrm{p}}$, as a function of time, $t$, to be

$m_{\mathrm{p}}(t)=M_{\mathrm{p}} \times \begin{cases}\sin ^{2}\left(\pi t / 2 T_{\text {growth }}\right) & t \leq T_{\text {growth }}, \\ 1 & t>T_{\text {growth }},\end{cases}$

where $M_{\mathrm{p}}=m_{\mathrm{p}}\left(T_{\text {growth }}\right)$ is the planet's final mass and $T_{\text {growth }}$ is the planet's growth time-scale. We do not allow gas to accrete on to the planet. To avoid numerical issues that arise when mass accumulates within the planet's Hill sphere, we slowly remove this material following the prescription used by Kley (1999). The total mass removed over an entire simulation is always less than 5 per cent of the planet's final mass.

\subsubsection{Boundary conditions}

At the radial boundaries of the disc, we utilize wave-killing zones (e.g. de Val-Borro et al. 2006) for $r \in[1,1.25] r_{\text {in }}$ and $r \in[0.84,1] r_{\text {out }}$ to damp the density and velocity fields in these regions towards the initial conditions for both the gas and the dust. We rapidly damp the outer zone on a time-scale of 1/500th of the outermost orbital period so that this region is mostly unperturbed. The inner region is damped at a slower rate of $1 / 3$ rd of the innermost orbital period. We use periodic boundary conditions in the azimuthal direction.

\subsubsection{Simulation suite}

We conduct a total of eight simulations in our primary study, encompassing planets with two different growth time-scales $\left(T_{\text {growth }}=10\right.$ and $\left.750 T_{\mathrm{p}}\right)$ and dust particles with four different sizes $(s=0.3 \mathrm{~mm}$, $1 \mathrm{~mm}, 3 \mathrm{~mm}, 1 \mathrm{~cm}$ ). The largest grain size corresponds to an initial Stokes number, $\mathrm{St}_{0}$, of 0.076 at the location of the planet. For each particle size, we explore the differences in the contrast and azimuthal profiles of the dust vortices that form in each case, with a focus on the distinctive characteristics of the corresponding synthetic images at typical ALMA beam sizes.

\subsection{Synthetic images}

We generate synthetic intensity images from our simulations at a cadence of one output per orbit by calculating a map of the flux contributions from the dust surface density in each grid cell over a range of grain sizes, a similar method to that used in other studies (e.g. Bae et al. 2016). Combining dust density fields is non-trivial since the gas vortices do not perfectly overlap between different simulations. These deviations result from the different adaptive time-steps used by FARGO for different grain sizes. To align the density fields for different grains, we rotate them so that each gas vortex aligns with the one from the simulation of the intermediate $3 \mathrm{~mm}$ grains. We find the proper alignment angle by testing all integer angles between 0 and $360^{\circ}$ and choosing the one with the lowest absolute difference in mass when subtracting each density field from the reference gas vortex at the same timestep. We checked the alignment manually and found this method is typically precise to within $5 \mathrm{deg}$, often less. This level of precision is sufficient, given the relatively large beam sizes in the convolution step. With this alignment procedure, we can then combine the dust density grids corresponding to different grain sizes.

We combine the density maps from the four sizes that we simulated into a single composite density map with a number density distribution of $n=n_{0}\left(s / s_{0}\right)^{-p}$, where $p=3.0$ and $n_{0}$ normalizes the initial gas-to-dust ratio to 100 . Such a high power-law index assumes significant grain growth in the vortex (Birnstiel, Dullemond \& Brauer 2010). The maximum grain size is assumed to be $1 \mathrm{~cm}$. In accordance with that power law, the weights applied to the density maps are as follows: 45 per cent to $1 \mathrm{~cm}, 37$ per cent to $3 \mathrm{~mm}, 12$ per cent to $1 \mathrm{~mm}$, and 5.3 per cent to $0.3 \mathrm{~mm}$. A lower power-law index of -3.5 would reduce the magnitude of the peak in the resulting images, as smaller grains exhibit flatter dust distributions.

The flux $F$ at a frequency $v$ is computed in each cell as

$F_{v}(r, \phi)=I_{v}(r, \phi) \frac{r \delta r \delta \phi}{d^{2}}$,

where $\delta r$ and $\delta \phi$ are the cell widths and the intensity $I_{v}$ is given by

$I_{v}(r, \phi)=B_{v}(T)\left[1-\mathrm{e}^{-\tau_{\nu}}\right]$.

The optical depth $\tau_{v}$ is

$\tau_{v}=\Sigma_{\text {dust }}(r, \phi ; s) \kappa_{v}$,

where $\kappa_{v}$ is the opacity of a particular-size grain when observed at a particular frequency. These opacities are computed from the Jena data base ${ }^{2}$ using Mie theory (Bohren \& Huffman 1983), assuming that the grains are magnesium-iron silicates (Jaeger et al. 1994; Dorschner et al. 1995).

The images are calculated at $\lambda=0.87 \mathrm{~mm}$, which corresponds to ALMA Band 7. The distance to the system is set to $d=140 \mathrm{pc}$. To incorporate the effects of finite resolution found in observations, we convolve the images with a range of beam diameters from 0.071 to 0.284 arcsec, corresponding to scales of $10-40$ au in the disc. Since most of the transition discs with observed asymmetries have large cavities, we neglect the inner disc $\left(r<r_{\mathrm{p}}\right)$ to better mimic existing observations and to prevent the inner region from contaminating the outer disc when convolving with large beam sizes.

We scale our simulations by placing the planet at $r_{\mathrm{p}}=20 \mathrm{au}$. We also use a temperature profile of $T=T_{0}\left(r / r_{\mathrm{p}}\right)^{-1}$ consistent with our hydrodynamic simulations, where $T_{0}$ is set by the scale height of the disc and assumes a mean molecular weight of $\mu=2.34$. The temperature of the star is set to $T_{\odot}=5770 \mathrm{~K}$. The disc is viewed face-on with an inclination of $i=0^{\circ}$.

In our analysis of the synthetic images, we measure the offset of the peak from the centre of the vortex directly from the image assuming no knowledge of the underlying gas vortex. We identify the edges of the vortex by choosing a fixed threshold relative to the intensity peak. From there, we assume the geometric centre is at the mid-point of the edges.

\footnotetext{
${ }^{2}$ from the Astrophysical Laboratory Group of the AIU Jena: http://www.as tro.uni-jena.de/Laboratory/Database/databases.html.
} 

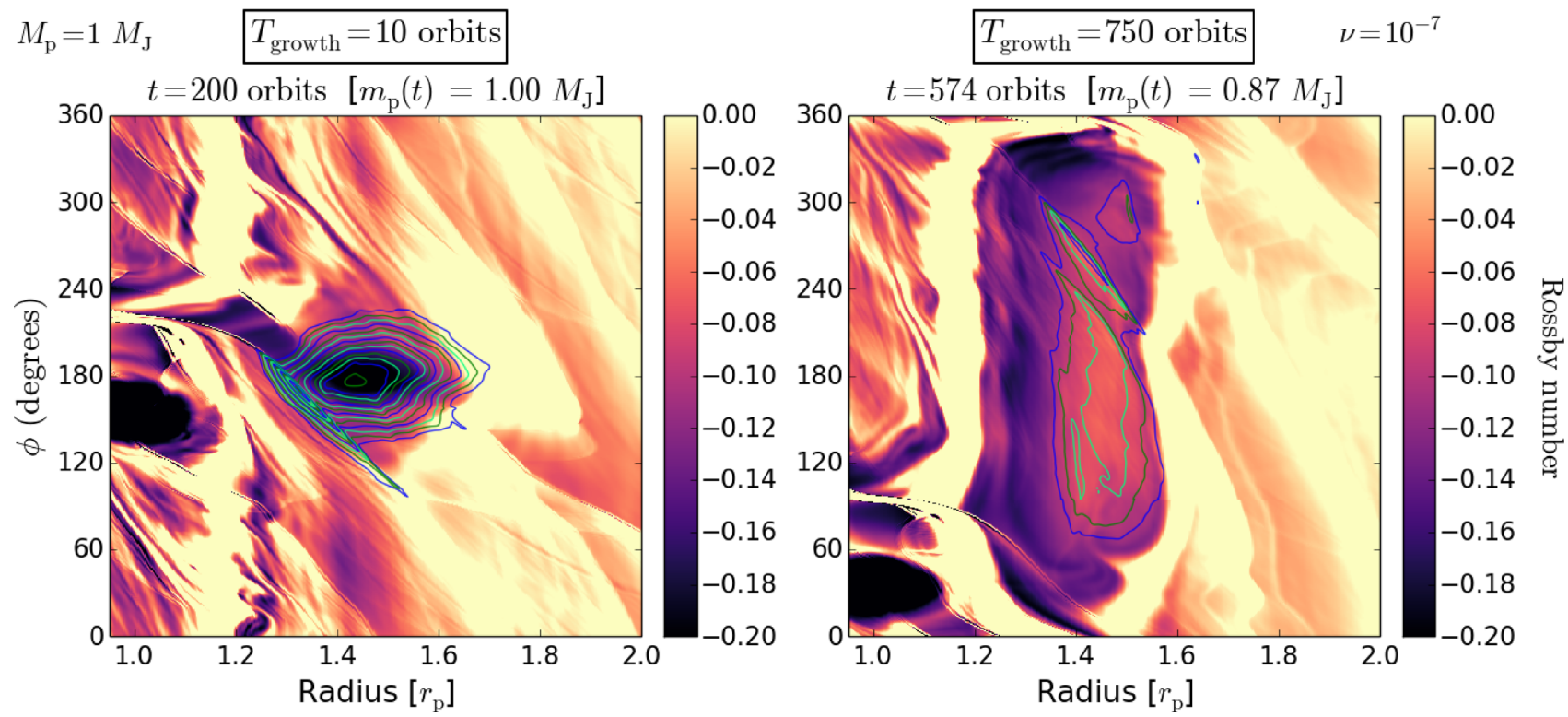

Figure 1. Rossby number snapshots of the concentrated vortex $\left(T_{\text {growth }}=10\right.$ planet orbits; left-hand panel $)$ and the elongated vortex $\left(T_{\text {growth }}=750\right.$ planet orbits; right-hand panel). Gas density contours are overlaid on each plot corresponding to $\Sigma_{\mathrm{g}} / \Sigma_{\mathrm{g}, 0}=1.0,1.1,1.2, \ldots, 2.3$. The concentrated vortex has a vorticity minimum at the centre and a smooth pressure bump, both of which facilitate dust trapping at the centre. The elongated vortex lacks these features, leaving the dust it traps much more spread out in the azimuthal direction. The Rossby number is defined as Ro $\equiv\left[\nabla \times\left(v-v_{\mathbf{K}}\right)\right]_{z} / 2 \Omega$, where $v$ is the gas velocity and $v_{\mathbf{K}}$ is the Keplerian gas velocity.

\section{RESULTS}

\subsection{Overview}

We find that elongated vortices do not trap dust in the same manner as concentrated vortices, making them easy to distinguish in synthetic images with sufficient resolution. It is widely accepted that concentrated vortices trap dust in pressure maxima at their centres (Barge \& Sommeria 1995; Tanga et al. 1996; Johansen, Andersen \& Brandenburg 2004), provided that they satisfy the condition $|\delta \mathbf{u}| \geq c_{\mathrm{s}}^{2} / 2 v_{\mathrm{K}}$, where $\delta \mathbf{u}$ is the velocity perturbation, $v_{\mathrm{K}}$ is the Keplerian velocity, and $c_{\mathrm{s}}$ is the sound speed. These pressure maxima also correspond to vorticity minima. Additionally, this relation assumes that a vortex maintains geostrophic balance between the pressure gradient and Coriolis force (see review by Lovelace \& Romanova 2014, and references therein).

On the contrary, elongated vortices do not have the same type of uniform structure (see Fig. 1). While they maintain a sharp pressure gradient towards their boundaries, their interior pressure profiles are mostly flat. The azimuthal gradient is negligible except near the edges, while the radial gradient also flattens out near the centre over a wider radial range than in the concentrated case. Without steeper pressure gradients, no vorticity minimum arises at the centre. Moreover, the interior profiles are ever-changing due to the repeated interactions between the vortex and the planet's spiral density waves. These waves act as a larger perturbation $\delta \Sigma / \Sigma_{\mathrm{g}}$ through the vortex in the elongated case. They also have a more pronounced effect of altering the overall structure of the gas in the elongated case compared to the concentrated case, which we suspect is due to shocks. As a result of its flatter and time-variable structure in the gas, an elongated vortex traps dust away from its centre, unlike a more idealized concentrated vortex.

As usual, the dynamics of dust particles in an elongated vortex are strongly dependent on particle size. Instead of concentrating at the centre, dust of all sizes circulates around the vortex on time- scales of a few dozen orbits. Larger dust particles concentrate more and circulate closer to the vortex centre. Only the largest particles ( $s \geq 3 \mathrm{~mm}$ ) exhibit pronounced peaks, while smaller dust particles $(s \leq 1 \mathrm{~mm})$ are spread out across nearly the entire vortex over a width of $\geq 180^{\circ}$. Even though larger dust particles are still the most concentrated in elongated vortices, they are also much more spread out compared to dust of the same size in concentrated vortices (see Fig. 2). Furthermore, dust preferentially circulates towards the front of the vortex in the direction of the planet's orbit, possibly due to the motion of the spiral density waves. The circulation of the dust as well as the interactions with the spiral waves makes the azimuthal profiles of elongated vortices highly variable over time.

The circulation of the dust across an elongated vortex produces a variety of signatures that distinguish it from a concentrated vortex. These include

(i) wide azimuthal extents

(ii) off-centre peaks, and

(iii) a skewness (lack of symmetry) about the peak, as well as

(iv) double peaks in newly formed vortices.

Observations with a beam size equal to or less than the semimajor axis of a putative planet - that is, a beam size much smaller than the radial location of the vortex itself - are needed to identify these features. With larger beam sizes, the edge of the vortex begins to blend with the background. As a result, it is difficult to find the azimuthal edges of the vortex, which in turn prevents precise measurements of the azimuthal extent as well as the magnitude of the peak offset, the latter of which is easily underestimated at lower resolution.

\subsection{Dust vortices}

As the dust circulates around an elongated vortex, the azimuthal extents of different-sized grains do not stay fixed over time (see Fig. 3). This behaviour is very different from that of concentrated vortices, 

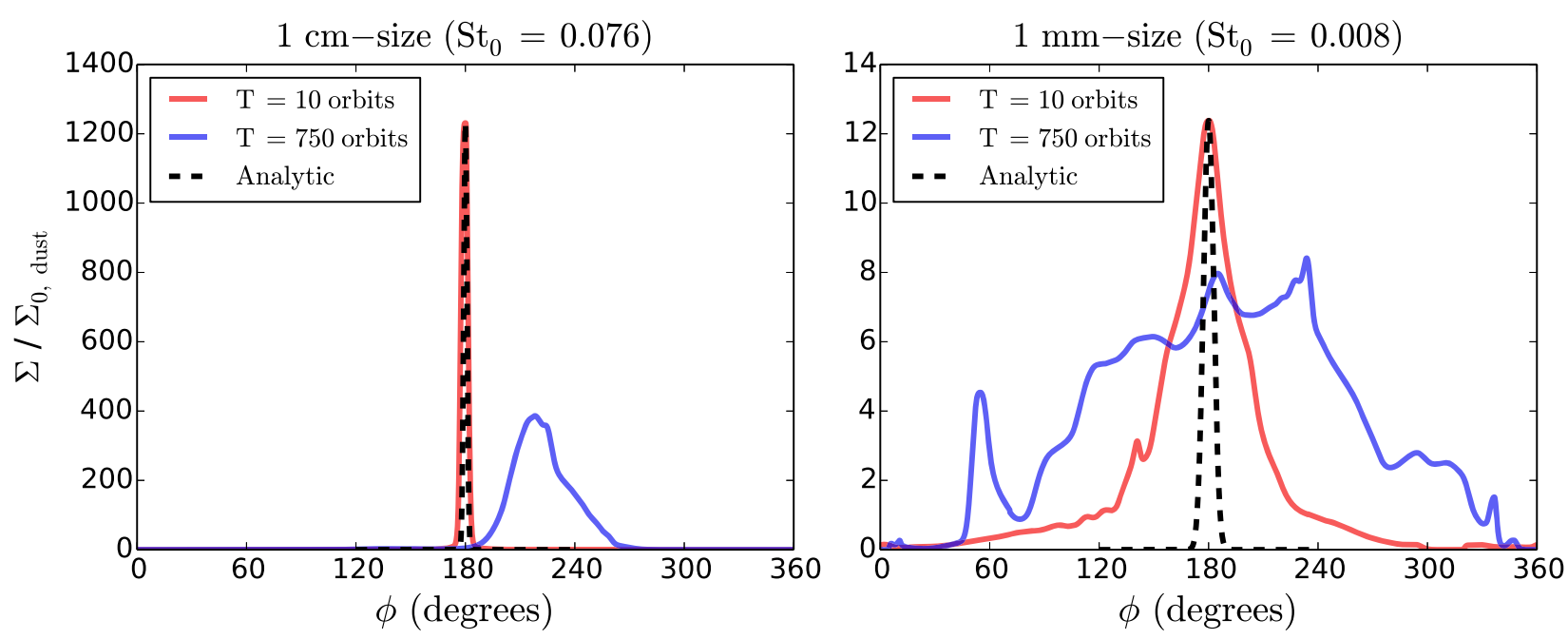

Figure 2. Central azimuthal dust density profiles of the concentrated vortex $\left(T_{\text {growth }}=10\right.$ planet orbits; shown in red $)$ and the elongated vortex $\left(T_{\text {growth }}=\right.$ 750 planet orbits; shown in blue) for cm-sized (left-hand panel) and mm-sized dust (right-hand panel). Analytic models (black dashed lines; see Section 3.2.1) through the centre are shown for comparison. For both grain sizes, the concentrated vortices trap dust in Gaussian profiles in the vortex centre. In contrast, the elongated vortices have off-centre peaks and much wider profiles. Snapshots are the same as in Fig. 1.

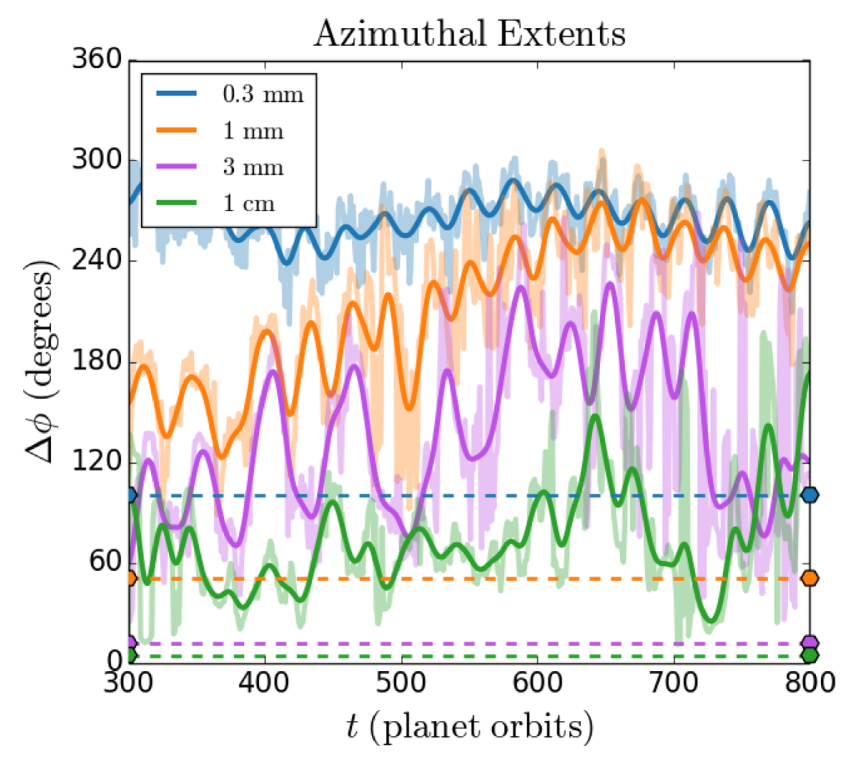

Figure 3. Extents of the elongated vortex over time for four different grain sizes. The extents of the concentrated vortex at a single time of $t=200$ are denoted with dashed lines for comparison.

where the extents hardly change. Furthermore, larger grains in elongated vortices are not necessarily much more concentrated than smaller grains. The extents of a concentrated vortex are roughly $60^{\circ}$ for $\mathrm{mm}$ grains, and less than $10^{\circ}$ for $\mathrm{cm}$ grains. There are also clear gaps between any two successive grain sizes from our simulations. In contrast, the extents of an elongated vortex vary between $60^{\circ}$ and $120^{\circ}$ for $\mathrm{cm}$ grains, and are between $90^{\circ}$ and $210^{\circ}$ for $3 \mathrm{~mm}$ grains. While the extent of the $1 \mathrm{~mm}$ grains is initially much narrower than that of the $0.3 \mathrm{~mm}$ grains by more than $90^{\circ}$, they both converge to a little above $240^{\circ}$. There are also rare instances where the $3 \mathrm{~mm}$ grains are slightly more concentrated than the $1 \mathrm{~cm}$ grains or slightly less concentrated than the $1 \mathrm{~mm}$ grains. These extents are measured with a threshold of 40 per cent of the peak; other choices do not change the qualitative results.
The circulation of the dust in an elongated vortex is also marginally size-dependent (see Fig. 4). Each period of circulation is roughly a few to several dozen orbits; however, the dust density peak rarely completes a closed trajectory around the vortex. These open trajectories are due to the narrowness of the vortex's radial extent and the non-uniformity of the gas in the vortex interior, the latter of which is partially attributed to shocks from the planet's spiral density waves. These features make it difficult to quantify the discrepancies between sizes. None the less, with larger grains collecting closer to the centre in both the radial and azimuthal directions, the peaks of different-sized grains do not perfectly overlap. When the vortex forms, the peaks are initially aligned. However, as the gas structure evolves, small differences in the peak locations propagate over time. Nevertheless, the offset between peaks at different grain sizes does not continue to increase indefinitely, which we suspect is due to the spiral waves inhibiting the motion of the dust towards the tail of the vortex. The small azimuthal discrepancies between these peaks flatten out the intensity peak in the resulting synthetic images.

The telltale signature of dust circulation in an elongated vortex is the movement of the peak away from the centre of the vortex. Only the dust density peaks for the largest grains $(s \geq 3 \mathrm{~mm}$ ) stand out. These peaks are shifted towards the front of the vortex more than half of the time. In particular, once the peak for the $\mathrm{cm}$ grains moves towards the front of the vortex, it can stay circulating at the centre or ahead for several hundred orbits. In contrast, on the rare occasions when the peak for these grains moves towards the back of the vortex, it will circulate back to the centre or the front within 20 orbits. We interpret this behaviour as evidence that the spiral density waves are helping carry the dust towards the front of the vortex in general. Peak offsets more than $30^{\circ}$ ahead of centre are common, while the largest offsets can exceed $60^{\circ}$. In comparison to other causes of offsets, the observed shifts exceeding $30^{\circ}$ are larger than those that might arise due to self-gravity and the indirect force for all particles with $s<5 \mathrm{~cm}$ (Baruteau \& Zhu 2016), as well as those caused by dust feedback (Barge et al. 2017). We cannot conclude whether incorporating dust feedback would enhance or diminish these effects, as that would depend on whether it induces azimuthal drift towards or away from the centre of the vortex. 


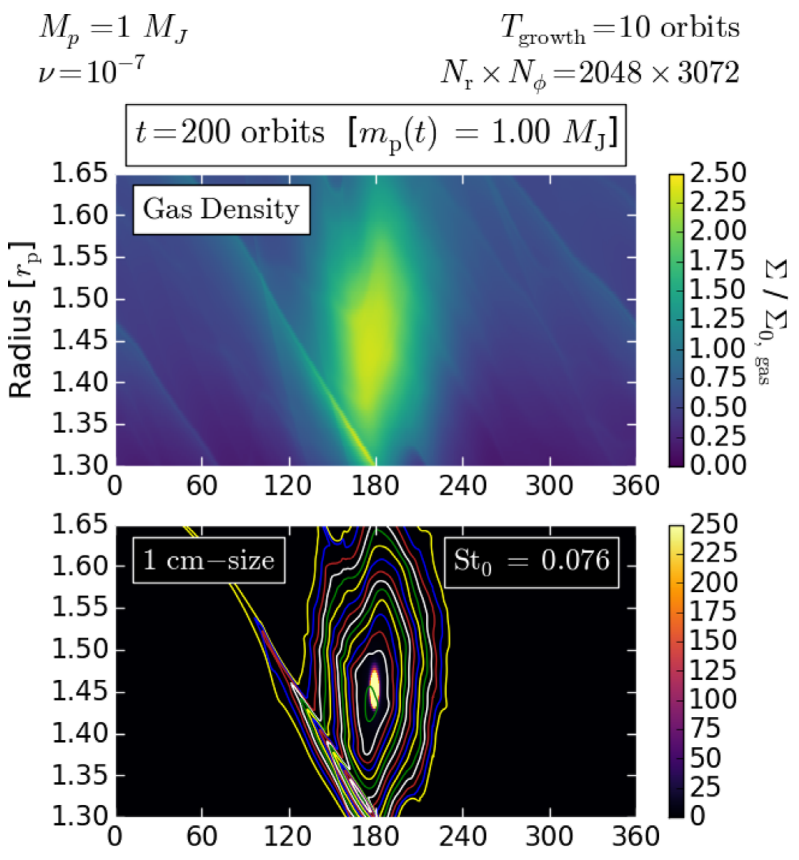

$$
\begin{array}{lr}
M_{p}=1 M_{J} & T_{\text {growth }}=750 \text { orbits } \\
\nu=10^{-7} & N_{\mathrm{r}} \times N_{\phi}=2048 \times 3072
\end{array}
$$
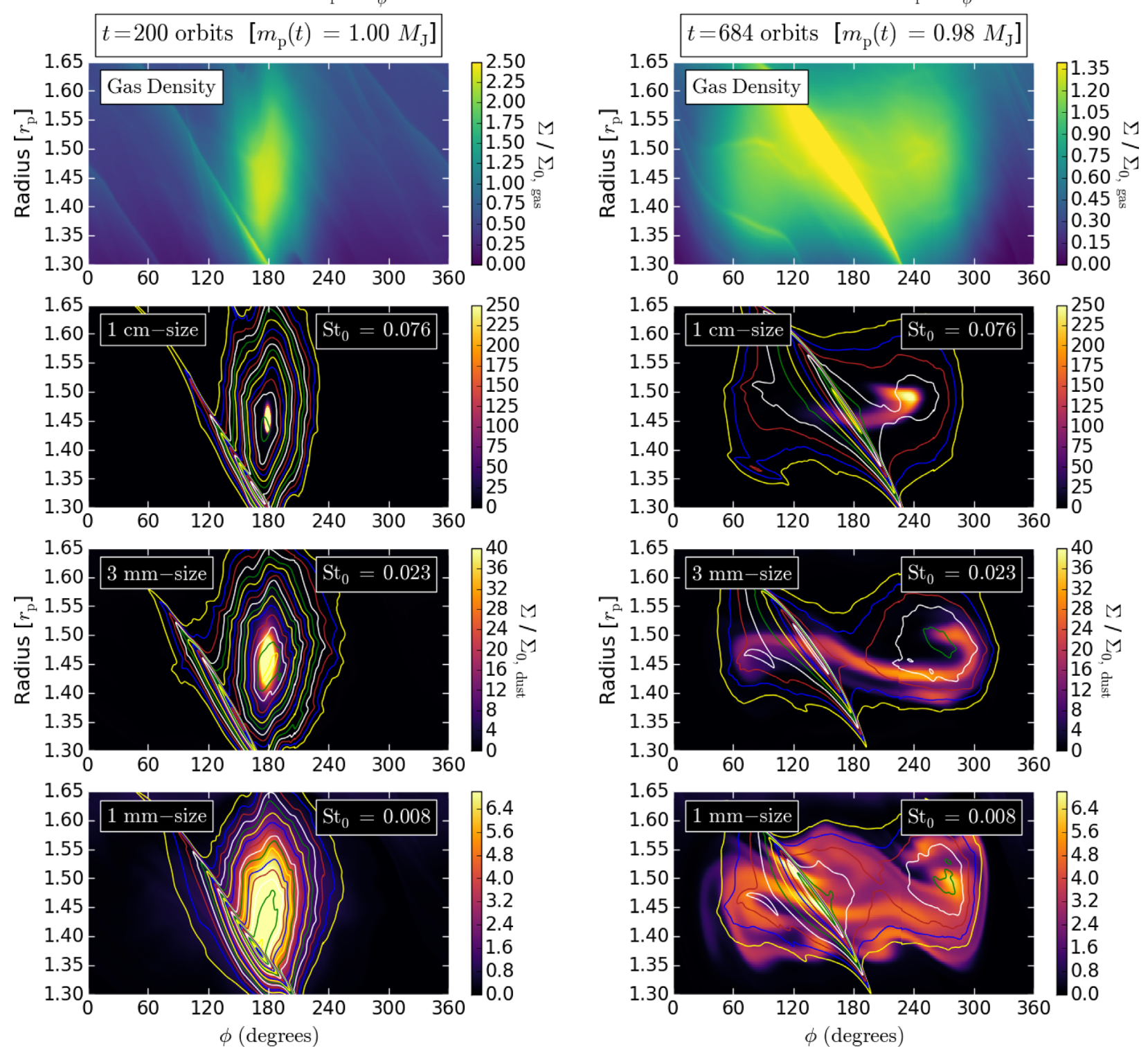

Figure 4. Density maps of the concentrated vortex $\left(T_{\text {growth }}=10\right.$ planet orbits; left-hand panels $)$ and the elongated vortex $\left(T_{\text {growth }}=750\right.$ planet orbits; right-hand panels). The top frame shows the gas distribution; the bottom frames show the dust distributions for the three largest grain sizes in our study: $1 \mathrm{~cm}$, $3 \mathrm{~mm}$, and $1 \mathrm{~mm}$. Gas density contours are overlaid on each dust density map corresponding to $\Sigma_{\mathrm{g}} / \Sigma_{\mathrm{g}, 0}=0.9,1.0,1.1, \ldots, 2.3$. In the elongated vortex, all three dust maps show dust circulating back towards the centre from the front of the vortex. Larger grains are more concentrated in the peak and have narrower extents. Movies are also included.

Even though the elongated vortex has less pronounced peaks than the concentrated vortex, it still has dust-to-gas ratios of the order of unity. In Fig. 4, the composite density field of the concentrated vortex achieves $\Sigma_{\mathrm{d}} / \Sigma_{\mathrm{g}}=5.8$, while the elongated vortex achieves a lower $\Sigma_{\mathrm{d}} / \Sigma_{\mathrm{g}}=1.6$. On longer time-scales, both vortices continue to accumulate dust unimpeded as the dust drifts inwards through the disc, although the magnitude of the peak in the elongated vortex increases at a much slower rate than in the concentrated vortex. With these high dust concentrations $\left(\Sigma_{\mathrm{d}} / \Sigma_{\mathrm{g}}>1\right)$, dust feedback may reduce the lifetimes of the vortices or further widen their azimuthal extents (Fu et al. 2014; Crnkovic-Rubsamen, Zhu \& Stone 2015), effects that we leave to future studies to investigate.

\subsubsection{Analytic dust-trapping model}

In Hammer et al. (2017), we used the analytic dust-trapping models of Lyra \& Lin (2013) to estimate dust profiles in both concentrated and elongated vortices. Perhaps unsurprisingly, our dust simulations show wild departures from the analytic models in the elongated case. This is consistent with their messier internal velocity and density structures, which do not feature the closed pressure contours and streamlines of Kida vortices (Kida 1981). To illustrate the discrepancy, we nevertheless compare our simulations with the analytic models below. Kida vortices are characterized by a constant aspect ratio, which we estimate as

$\chi=r \Delta \phi / \Delta r$, 
where $r \Delta \phi$ and $\Delta r$ are the azimuthal and radial extents of the gas vortex, respectively, which we measure from our simulations.

We then use Lyra \& Lin's model for the dust distribution across a vortex given by

$\rho_{\text {dust }}(a)=\rho_{0} \exp \left\{-\frac{a^{2} f^{2}(\chi)}{2 H^{2}}(S+1)\right\}$

(see their equation 64) to derive the expected azimuthal profile. Here, $a$ is the semiminor axis of an elliptical density contour inside the vortex; $\rho_{0}$ is the peak dust density in the vortex at a particular orbit in the simulation; $S=\mathrm{St} / \delta$ is the ratio of the particles' Stokes number to the dimensionless diffusion coefficient in the vortex; and $f(\chi)$ is a scale function of the order of unity (see their equation 35). Unlike in Hammer et al. (2017), we select $\delta=3 \times 10^{-5}$ to be the local value of the $\alpha$-viscosity, taken from our simulations. We do not presume any additional turbulence due to the presence of the vortex since we did not add any extra turbulence in the simulation.

In Fig. 2, we compare the azimuthal density distributions at the radial centre of the vortex for dust with $s=1 \mathrm{~cm}$ and $1 \mathrm{~mm}$ to the analytic models of the concentrated vortex. (With the dependence on $\chi$ in equation (11), the analytic profiles of the elongated vortex are even narrower than those of the concentrated vortex shown in Fig. 2.) We find that the concentrated vortices have Gaussian profiles that match up rather well with the analytic profiles, aside from being slightly wider than predicted. Interestingly, the analytic models for the two largest grain sizes would very closely agree with the simulations if they assumed a factor of 5 higher level of diffusion $\left(\delta=1.5 \times 10^{-4}\right)$ compared to the prescribed value used in the simulations.

On the other hand, the profiles in the elongated vortices disagree qualitatively and quantitatively. The profiles for the $\mathrm{cm}$-sized grains have much wider peaks that lack the symmetry of a Gaussian, while all other smaller simulated grain sizes have much flatter profiles. As a result, we advocate caution in applying analytic models to non-ideal vortices.

\subsection{Synthetic images}

The circulation of dust in an elongated vortex creates several signatures in our synthetic images, the most notable of which is the off-centre peaks that are naturally accompanied by a skewness in the azimuthal profiles. The extent can also distinguish an elongated vortex from a concentrated one, provided that it is possible to identify the vortex edges. Identifying the largest peak offsets and measuring the wider azimuthal extents both typically require a beam size equal to or less than the planet's semimajor axis - that is, roughly two-thirds the radial location of the peak in the asymmetry (see Fig. 5).

The largest offsets of more than $30^{\circ}$ are regularly identifiable with this high of a resolution. Fig. 6 shows the distribution of peak offsets for four different beam diameters: 10, 20, 30, and $40 \mathrm{au}$ - where the planet is located at $20 \mathrm{au}$. With a beam diameter of the planet's separation (1.0 $r_{\mathrm{p}}$; equivalent to $\left.0.14 \mathrm{arcsec}\right)$, shifts of more than $30^{\circ}$ in either direction occur 30 per cent of the time. This number drops to 10 per cent for a diameter of 30 au $\left(1.5 r_{\mathrm{p}}\right.$; equivalent to the separation of the vortex) and less than 2 per cent for twice the planet's semimajor axis. Relatively large shifts of $>20^{\circ}$ still appear in 60 per cent of snapshots for a beam size of the planet's separation, a number that drops to 20 per cent for a beam of the vortex's separation. However, we caution that the offsets in this more moderate range could also be attributed to the indirect force without self-gravity, an effect that yields maximum shifts of $25^{\circ}$ (Baruteau \&
Zhu 2016). Additionally, larger beam sizes can be insufficient for resolving secondary peaks (see Section 3.3.1). For these reasons, we recommend a beam size of at most the planet's separation to measure the peak offset instead of the vortex's separation or larger.

High resolution is needed to measure a vortex's azimuthal extent as well. The bottom panels of Fig. 5 show the azimuthal profile across the radial centre of a vortex for a single snapshot that is observed with a range of beam sizes from 10 to 40 au, as with Fig. 6 . With higher resolution, the sharpness of the edges makes it possible to identify their location with a good choice of threshold. Lower resolution at or above the separation of the vortex blends the edges with the background too much to pinpoint their location. This lack of precision makes it difficult to tell if the vortex has an elongated azimuthal extent, and is also the reason for the lower resolution underestimating the magnitude of the peak offsets. Moreover, the concentrated vortex also starts to appear elongated at these resolutions. We also caution that observations of discs that are not face-on may require even higher resolution to measure a vortex's azimuthal extent.

The appearance of the azimuthal profiles is dependent on the choice of grain size distribution. We can estimate the contributions of the grain sizes by calculating the peak intensity after separately removing each grain size from the images. Leaving out the $1 \mathrm{~cm}$ grains results in an average of a 29 per cent reduction in the peak of the elongated vortex, while removing the $3 \mathrm{~mm}$ grains leads to a 30 per cent reduction, the $1 \mathrm{~mm}$ grains a 17 per cent reduction, and the $0.3 \mathrm{~mm}$ grains a 23 per cent reduction. With their sharper distributions and higher contribution levels, the $1 \mathrm{~cm}$ and $3 \mathrm{~mm}$ grains are therefore most responsible for the properties of the peak. The azimuthal profiles of a vortex with a flatter grain size distribution such as $p=3.5$ would be more dominated by smaller grains near $s \approx 3 \mathrm{~mm}$. Furthermore, we note that the relatively jagged azimuthal intensity profile shown in the right-hand panel of Fig. 5 for the smallest beam size might appear smoother had we simulated a more continuous distribution of grain sizes. Additionally, a continuous size distribution might also lead to vortices frequently having flatter intensity peaks due to the slight differences in the shifts of different-sized grains.

The circulation of dust in an elongated vortex can lead to quick changes in the azimuthal profiles of a vortex. Even though the circulation period itself is very slow, factors such as interactions with the planet's spiral density waves, the spreading of the peak, and dust motion near the azimuthal edges can cause significant variability within a single orbit. As a result of these effects, the location of the peak relative to the vortex centre changes by more than $15^{\circ}$ in 18 per cent of snapshots taken at successive planet orbits with a beam diameter of $10 \mathrm{au}\left(0.5 r_{\mathrm{p}}\right.$; equivalent to $\left.0.07 \mathrm{arcsec}\right)$. It may be possible to observe these changes in vortices induced by planets in the inner disc on time-scales of the order of decades. None the less, a lack of variability is still more common, with 42 per cent of successive snapshots having the peak unchanged to within $5^{\circ}$.

\subsubsection{Double-peaked structures}

When a vortex initially forms, it can have a very different doublepeaked structure as a signature. This structure arises from the lag between the dust vortices merging relative to the gas vortices. As the higher-number modes merge into a single $m=1$ mode, they are still spread out in azimuth (see the left-hand panel of Fig. 7). This larger azimuthal extent of the end-product vortex prevents the separate peaks of the previously co-orbital vortices from naturally 

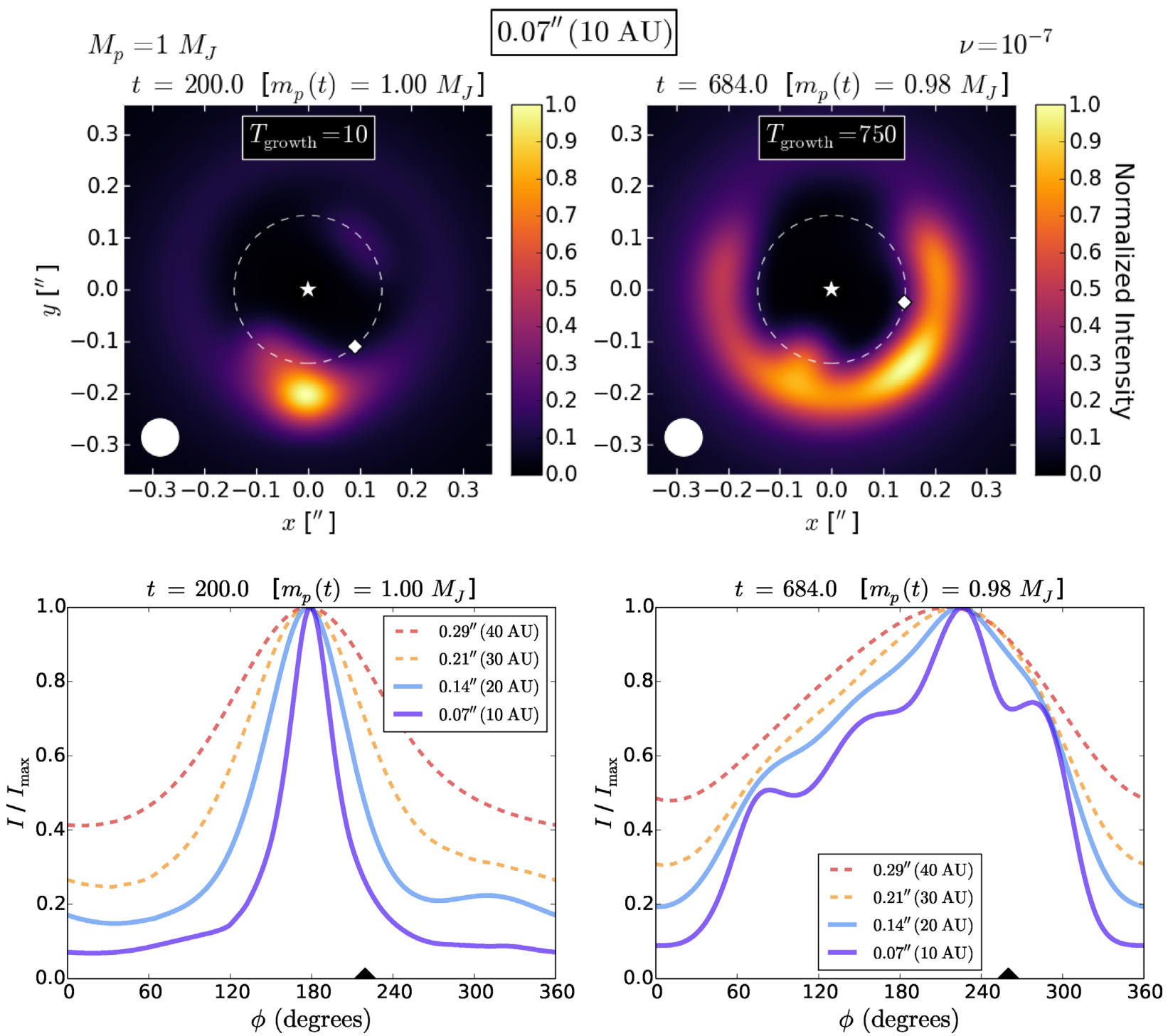

Figure 5. Top: Synthetic images of the concentrated vortex $\left(T_{\text {growth }}=10\right.$ planet orbits; left-hand panels $)$ and the elongated vortex $\left(T_{\text {growth }}=750\right.$ planet orbits; right-hand panels). Bottom: Comparison of central azimuthal intensity profiles for four different beam sizes. The black triangle indicates the location of the planet. With the smallest beam size, the peak in the elongated vortex is $40^{\circ}$ off-centre. Movies of both types of plots of the elongated vortex are also included.

merging into a single peak on a short time-scale, like they do in the concentrated case. Instead, the double-peaked structure persists for over 100 orbits for the $1 \mathrm{~cm}$ grains in our fiducial elongated vortex simulation. The smaller $3 \mathrm{~mm}$ grains only maintain this pattern for about 60 orbits.

In some snapshots, the double peaks of different-sized grains are aligned (as shown in the left-hand panels of Figs 7 and 8), while in others they can be far apart. When these peaks are not aligned, the resulting azimuthal intensity profiles are relatively flat due to the lack of a distinct peak location. It is also possible for the double-peaked signature to arise later in the vortex's lifetime (as shown in the right-hand panels of Figs 7 and 8); however, one of the peaks is typically dominant over the other in this case. The early-on double-peaked structure resembles the signature found by Baruteau \& Zhu (2016) for the largest grains in their study of vortices with self-gravity. Multiwavelength observations should be able to distinguish between these two scenarios, as the shifts in their study have a much steeper monotonic size dependence compared to those in our study, where the shifts of different-sized grains can still overlap and have less of a predictable pattern in general.

\subsubsection{Other features}

Besides the vortex exterior to the planet, the inner edge of the gap also triggers a vortex interior to the location of the planet. In the case of the elongated vortex, the slow growth of the planet gives the largest dust particles time to drift towards the inner edge of the disc while the planet itself prevents much of the dust in the outer disc from reaching the inner disc. As a result, the interior vortices should appear weaker than the exterior vortices due to the lower supply of the largest dust particles that attain the highest levels of concentration in vortices located in the outer disc.

Aside from the vortices that trap dust, the planet also collects dust at the L5 Lagrange point located $60^{\circ}$ behind its location in both cases of short and long growth times. As shown in Fig. 1, this point also corresponds to a local vorticity minimum. Since the rest of the gap is empty, this co-orbital dust could be visible in 


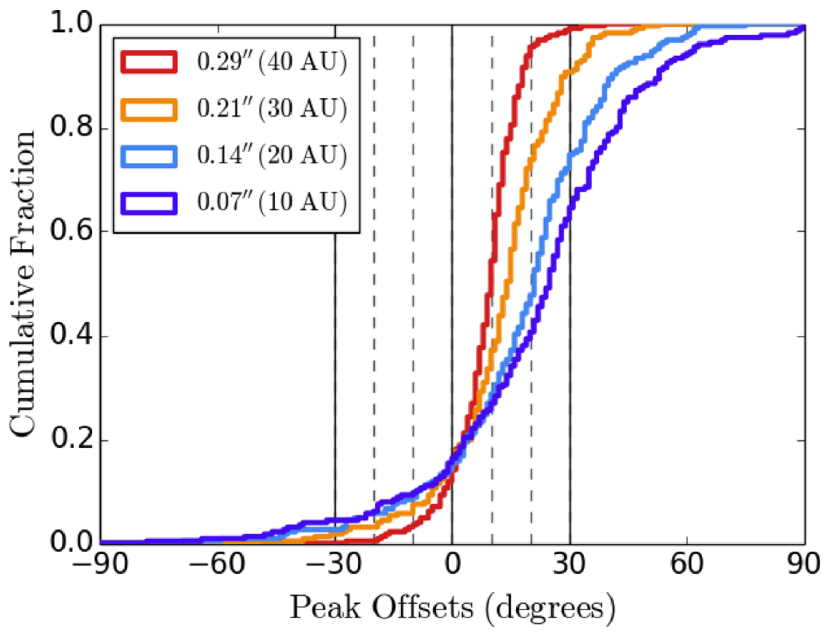

Figure 6. Histogram of the peak offsets in elongated vortices over time for four different beam diameters. Larger offsets are much more frequent with higher resolution.

observations, as shown in the top panels of Fig. 5 and the top right panel of Fig. 8. Detecting such a signature would help confirm that a vortex was indeed triggered by a planet and also reveal the planet's location.

\section{APPLICATIONS TO OBSERVATIONS}

\subsection{Confirmation of elongated planet-induced vortices}

To complement our criteria for determining the shape of a vortex, we suggest the following methods for assessing whether an observed asymmetry may be induced by a planet, rather than the boundary of a dead zone:

(1) Detecting trojan dust co-orbital with the planet: As stated in Section 3.3.2, an elongated planet-induced vortex should also be accompanied by dust at the L5 Lagrange point - that is, co-orbital with and $60^{\circ}$ behind the location of the planet.

Even with small beam sizes $(0.07$ arcsec $)$, this dust feature should overlap with the interior side of the vortex in the outer disc if it is located in the azimuthal range of the vortex (see the top right panel of Fig. 5). This feature is weaker and could be missing if the vortex is newly formed, which would suggest the planet is in the early stages of its runaway gas growth phase.

(2) Distinguishing from dead-zone boundary vortices: Previous studies of vortices generated by dead-zone boundaries have shown that they are also elongated (e.g. Lyra \& Mac Low 2012; Regály et al. 2012). We suggest two ways to distinguish them: First, planet-induced vortices frequently have off-centre peaks due to repeated perturbations from the planet's spiral arms. On the contrary, Regály, Juhász \& Nehéz (2017) find that without these interactions, vortices at dead-zone boundaries remain symmetric even when they have wide azimuthal extents.

Secondly, Regály et al. (2017) find that vortices at dead-zone boundaries are much more radially extended than those induced by rapidly grown planets. In contrast, we have shown that elongated vortices have similar radial extents than concentrated vortices, providing another distinguishing criterion.

(3) Distinguishing from dead-zone boundary vortices with feedback: In an alternative model with a longer simulation time and dust feedback, Miranda et al. (2017) find the dust structure of a dead zone is rather concentrated at the centre even though the gas structure is still very elongated.

Furthermore, in their 'GasHigh' run that is the most similar to our study, the primary concentration of dust at the centre breaks apart into several smaller clumps due to feedback and a much higher dust-to-gas ratio of $\Sigma_{\mathrm{d}} / \Sigma_{\mathrm{g}} \approx 10$. These secondary clumps spread out widely in azimuth, while the primary clump remains dominant (see their fig. 3c). We expect this pattern to produce multiple peaks at a given radial distance that would be easy to distinguish from the singular primary off-centre peaks that characterize our synthetic images in all but the initial stages of vortex formation.

It is possible that feedback may also cause elongated planet-induced vortices to break up into clumps. None the less, these multiclump planet-induced vortices should still be distinguishable from deadzone vortices. In the dead-zone vortex model from Miranda et al. (2017), there is hardly any dust between separate clumps since the vortex was originally concentrated before it broke apart. This is distinct from our study, in which the dust is always spread out across the entire extent of the vortex. Thus, we expect the presence of dust between adjacent peaks to be unique to elongated planet-induced vortices.

(4) Observing time variability in elongated vortices: A planet's spiral arms can change the dust structure of elongated vortices over time-scales as short as one-third of an orbit.

For a planet located at 5 au around a solar mass star, we may be able to see changes in the azimuthal structure of an observed asymmetry in about 3-4 yr if it were an elongated vortex - albeit not always. In our simulations, we only see a dramatic shift in the peak or the azimuthal profiles within a single orbit occurring about once every five orbits. Presently, no system has an asymmetry at a small enough separation to look for variability on these short time-scales.

\subsection{Comparisons to theoretical models}

(1) Modelling planet and disc parameters of vortices: There is widespread interest in inferring the masses and orbits of unseen planets from the features they create in their discs - most notably vortices (e.g. Bae et al. 2016), spiral arms (e.g. Dong et al. 2015), and gaps (e.g. Dipierro \& Laibe 2017). It is already difficult to constrain these properties with vortices since the characteristics of a vortex are degenerate between different combinations of planet masses, disc viscosities, and temperature profiles, as well as other factors (e.g. Fu et al. 2014). The shape of a vortex adds an additional complication.

The resolutions used to observe systems with asymmetries are not always sufficient to discern whether these features are concentrated or elongated (see Fig. 5). As a result, models that assume these vortices are concentrated may be underestimating the planet's mass or overestimating the disc viscosity, among other possibilities. For these systems, higher resolution images are needed to resolve the shapes of these asymmetries in order to provide better input for predicting the properties of these undetected planet candidates and their discs.

(2) Validating planet formation models: There are few good observational methods to constrain planet formation time-scales for giant planets. Our best constraints come from measuring the ages of stars in systems with planet candidates of this size (e.g. Sallum et al. 2015; Donati et al. 2016; Keppler et al. 2018) or the lifetimes of protoplanetary discs in general (e.g. Bell et al. 2013), both of which naturally overestimate the ages of the associated planets. The discovery of an elongated planet-induced vortex could offer 


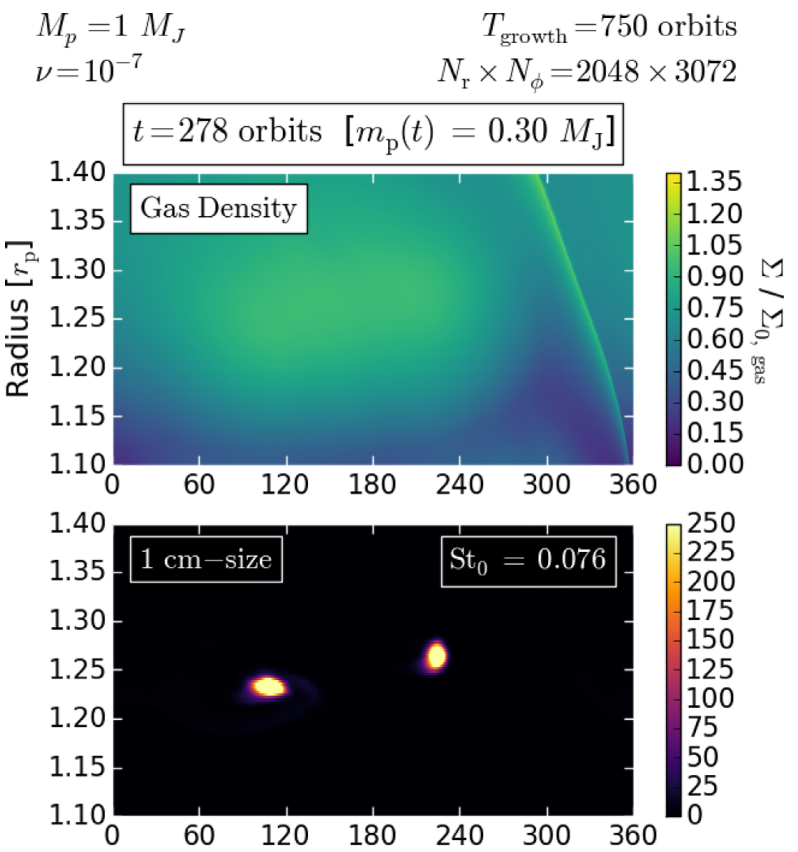

$$
\begin{array}{lr}
M_{p}=1 M_{J} & T_{\text {growth }}=750 \text { orbits } \\
\nu=10^{-7} & N_{\mathrm{r}} \times N_{\phi}=2048 \times 3072
\end{array}
$$
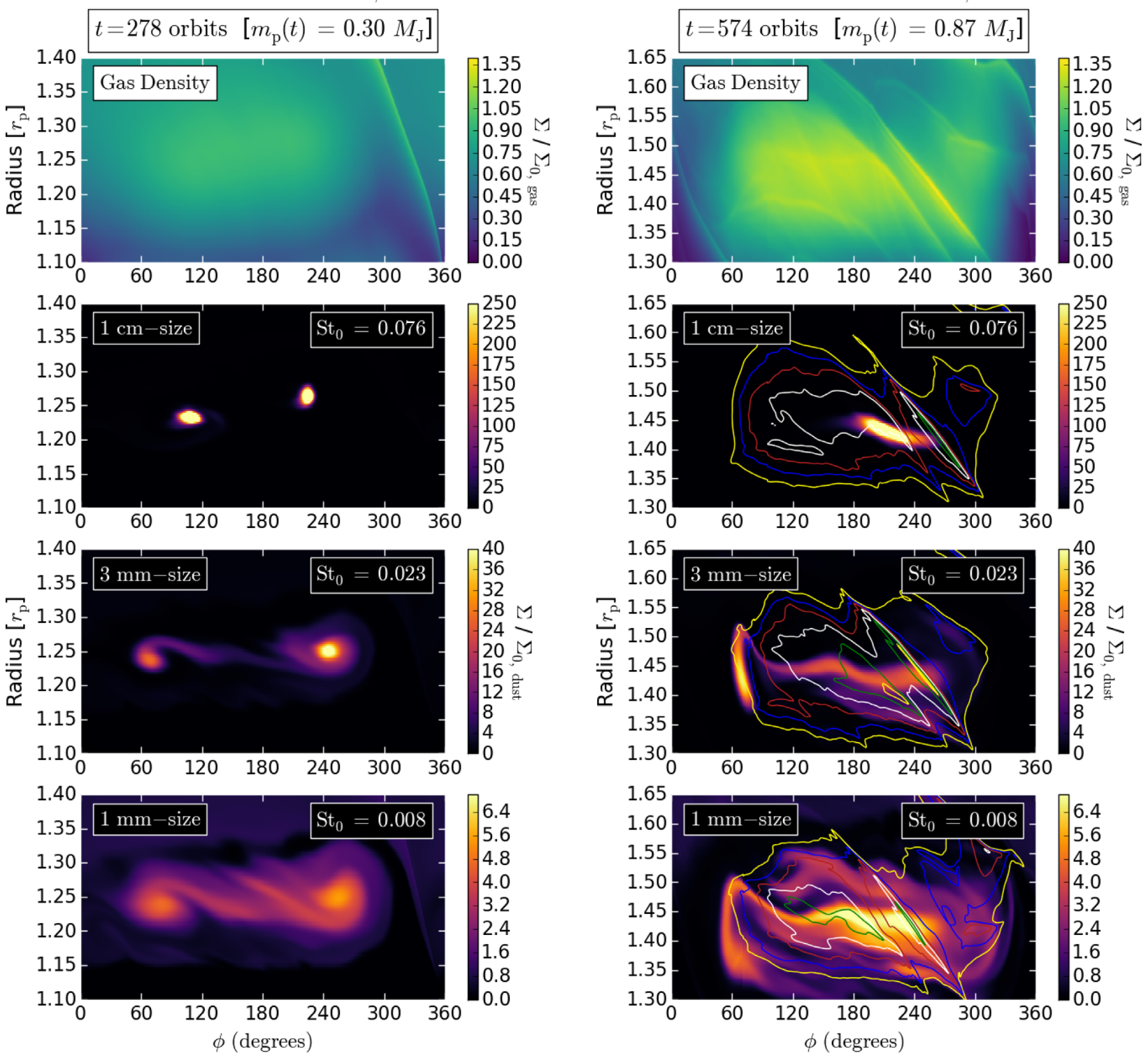

Figure 7. Density maps of the elongated vortex ( $T_{\text {growth }}=750$ planet orbits) that exhibit double peaks in the corresponding synthetic images. The top frame shows the gas distribution; the bottom frames show the dust distributions for the three largest grain sizes in our study: $1 \mathrm{~cm}, 3 \mathrm{~mm}$, and $1 \mathrm{~mm}$. The left-hand panels show an earlier snapshot $\left[t=278 ; m_{\mathrm{p}}(t)=0.30 M_{\text {Jup }}\right]$ within 50 orbits of the initial vortices merging into an $m=1$ mode. The right-hand panels show a later snapshot $\left[t=574 ; m_{\mathrm{p}}(t)=0.87 M_{\text {Jup }}\right]$ where the differences in the circulation patterns of the $1 \mathrm{~cm}$ grains and the other smaller grains have also created a double-peaked structure in the composite map.

some more direct insight into the process by which giant planets form.

First, it would show that the vortex was triggered while its accompanying planet was still growing. Additionally, it would help verify that gas giant planets take a relatively long time to complete the runaway gas accretion phase (at least a few hundred orbits for a Jupiter-mass planet and at least 1000 orbits for a 5 Jupiter-mass planet), as expected for the runaway growth phase of core accretion in a low-viscosity disc (Lissauer et al. 2009; Hammer et al. 2017). On the contrary, finding a concentrated asymmetry would not necessarily indicate a planet formed rapidly. It might instead suggest that the vortex did not form until after the planet finished growing, that other factors such as thermodynamic effects shaped the asymmetry
(Owen \& Kollmeier 2017), or that a planet may not be responsible for the feature in the disc.

\subsection{Vortex candidates}

In this subsection, we compare our synthetic images to observed discs with asymmetries. We find one good candidate system, HD $135344 \mathrm{~B}$, for harbouring an elongated planet-induced vortex. We also discuss two other systems - RY Lupus and T4 - where an elongated vortex cannot be ruled out as an explanation of their asymmetric features. Throughout this section, we presume that the asymmetries are located at a separation of $1.4 r_{\mathrm{p}}$ and that a beam size corresponding to $1.0 r_{\mathrm{p}}$ is needed to resolve each feature. 

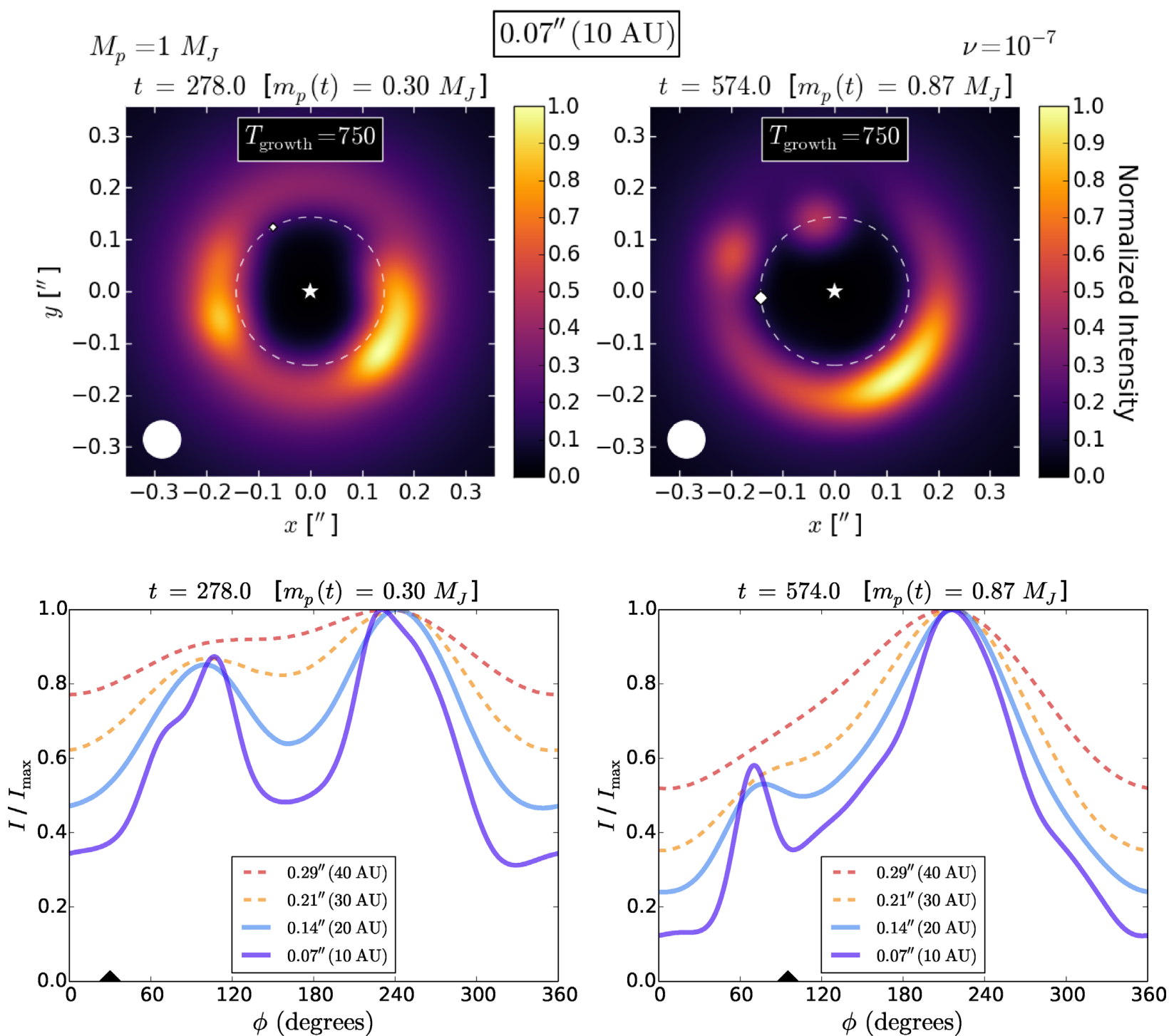

Figure 8. Top: Synthetic images of the elongated vortex $\left(T_{\text {growth }}=750\right.$ planet orbits $)$ that exhibit double peaks shortly after the vortex forms $\left[m_{\mathrm{p}}(t)=\right.$ $0.30 M_{\mathrm{Jup}}$; left-hand panels] and later on $\left[m_{\mathrm{p}}(t)=0.87 M_{\mathrm{Jup}}\right.$; right-hand panels]. At the later time, there is also a distinct signature of the co-orbital dust at L5 inside the vortex. Bottom: Comparison of central azimuthal intensity profiles for four different beam sizes. The black triangle indicates the location of the planet. There is a clear double-peak signature with a beam diameter of $<0.14 \operatorname{arcsec}$ in the earlier phase and $<0.07$ arcsec in the later phase.

There are not many good elongated vortex candidates in large part because most systems with asymmetric features have not been observed with sufficient resolution. Moreover, the wide separations of the majority of these features place them beyond the expected locations of giant planets (Bowler \& Nielsen 2018).

(i) $H D 135344 B$ is surrounded by a disc that includes a ring at $0.32 \operatorname{arcsec}(45 \mathrm{au})$ and an asymmetry at $0.54 \operatorname{arcsec}(75 \mathrm{au})$, leaving space for a potential planet near $0.38 \operatorname{arcsec}(54 \mathrm{au})$ in between the two features (van der Marel et al. 2016). It also exhibits spirals in scattered light (Muto et al. 2012; Garufi et al. 2013; Stolker et al. 2016). Unlike most other systems, its asymmetry has been observed with a small enough beam size to resolve that it has both a wide azimuthal extent a little under $180^{\circ}$ and a peak that is noticeably off-centre. Recent observations have confirmed the presence of a skewness about an off-centre peak as well as a decrease in the azimuthal extent at larger wavelengths (Cazzoletti et al. 2018), suggesting that the asymmetry could be an elongated planet-induced vortex. Without self-gravity in our simulations, we cannot test whether the observed spirals in the system could be caused by the vortex, as van der Marel et al. (2016) propose. We recommend future computational studies incorporate self-gravity to study this possibility.

(ii) $R Y$ Lupus has a disc that is inclined at $69^{\circ}$ from the line of sight. It features an asymmetry with an azimuthal extent of roughly $240^{\circ}$ located at $\approx 0.33 \operatorname{arcsec}(50 \mathrm{au})$ and a contrast of about 1.5 (Ansdell et al. 2016). Its azimuthal intensity profiles are relatively flat across the asymmetry. The system was observed with a beam size of 0.38 arcsec $\times 0.33$ arcsec. A beam diameter of at most 0.20 arcsec, if not smaller due to the system's high inclination, is needed to better assess the shape of the asymmetry.

(iii) $T 4$ (also known as $J 10563044$ ) has a relatively face-on disc containing an elongated asymmetry at $\approx 0.40 \operatorname{arcsec}(64 \mathrm{au})$ with an off-centre peak (Pascucci et al. 2016). The feature has an azimuthal extent of about $160^{\circ}$ and a very low contrast of $\sim 1.2$. With the relatively large beam size of $0.71 \operatorname{arcsec} \times 0.47$ arcsec, it is difficult 
to tell if the feature is elongated. A much smaller beam diameter of at most 0.24 arcsec is needed to examine this feature in detail.

\section{CONCLUSIONS}

We have shown that elongated vortices appear very distinct from concentrated ones in observations taken with beam diameters no larger than the planet's semimajor axis. We suggest that an asymmetry can be classified as an elongated vortex if it is characterized by the following criteria:

(i) a wide azimuthal extent of at least $180^{\circ}$,

(ii) an off-centre peak, and

(iii) a skewness (lack of symmetry) about the peak, or

(iv) double peaks (primarily in newly formed vortices).

As with standard Kida vortices, observations at different wavelengths can verify that the asymmetry is a dust-trapping vortex in general by showing that the feature is more concentrated at larger wavelengths due to the larger particles concentrating more narrowly in the azimuthal direction (van der Marel et al. 2015). However, the gap in azimuthal extents of different-sized grains can vary considerably. In rare instances, there may not be a noticeable gap between different-sized grains. At later times, smaller-sized grains ( $s$ $\leq 1 \mathrm{~mm}$ ) converge to a similar extent. Furthermore, other studies have found this signature may fade away if the gas vortex has already dissipated (Fuente et al. 2017; Surville \& Mayer 2018).

We offer several suggestions for how to interpret observations of asymmetries that may be elongated vortices. In particular:

(1) An elongated vortex induced by a planet may also be accompanied by trojan dust that is co-orbital with the planet at the L5 Lagrange point.

(2) We can distinguish an elongated vortex generated by a planet from one generated at a dead-zone boundary by observing a planetinduced vortex's off-centre peaks or by resolving the planet-induced vortex's much thinner radial extent.

(3) Another signature of elongated vortices is that the interaction between the planet's spiral density waves and its associated vortex could change the azimuthal intensity profiles of a vortex within a few years for a planet at Jupiter's separation from the Sun, even when the waves are not overlapping the vortex.

(4) If an asymmetry is wide enough to possibly be elongated, resolutions equal to or less than the planet's semimajor axis are needed to model the planet mass that is necessary to explain the asymmetry with a planet-induced vortex. Without this high of a resolution, it would be difficult to classify an asymmetry as concentrated or elongated and in turn make an accurate model of the vortex

(5) Discovering an elongated planet-induced vortex would help show that gas giant planets generate vortices during their runaway gas accretion phase while they are still forming. The existence of such a vortex would also show that this growth phase takes relatively long to complete (at least several hundred orbits or more, depending on the planet and disc parameters). In contrast, a concentrated asymmetry may favour an alternate explanation.

We propose that HD 135344B may harbour an elongated planetinduced vortex, as it contains an asymmetry with a wide azimuthal extent, more concentrated azimuthal extents at longer wavelengths, and an off-centre peak. However, follow-up modelling that incorporates the system's parameters would be needed to show further support for this idea. No other observed system has shown favourable signs that it contains an elongated vortex, in part due to the resolution used thus far in ALMA observations. We suggest RY Lupus as another system with an asymmetry that may be a good elongated planet-induced vortex candidate. Confirming this hypothesis would require observations taken with a beam diameter of the planet's assumed separation at $\approx 0.20 \mathrm{arcsec}$, if not higher resolution due to the system's high inclination.

\section{ACKNOWLEDGEMENTS}

We thank the referee for many helpful comments that greatly improved this manuscript. We thank Zhaohuan Zhu for providing the two-fluid FRAGO code. We would also like to thank Megan Ansdell, Paolo Cazzoletti, and Nienke van der Marel for helpful discussions on the candidate vortex images. Additionally, we thank Jeffrey Fung for discussions of the planet's co-orbital dust. $\mathrm{MH}$ is supported by the NSF Graduate Research Fellowship under Grant No. DGE 1143953. PP is supported by NASA through Hubble Fellowship grant HST-HF2-51380.001-A awarded by the Space Telescope Science Institute, which is operated by the Association of Universities for Research in Astronomy, Inc., for NASA, under contract NAS 5-26555. KMK is supported by the National Science Foundation under Grant No. AST-1410174. MKL is supported by the Theoretical Institute for Advanced Research in Astrophysics (TIARA) based in Academica Sinica Institute of Astronomy and Astrophysics (ASIAA). This work is also supported by NASA Astrophysics Theory Program grant NNX17AK59G. The El Gato supercomputer, which is supported by the National Science Foundation under Grant No. 1228509, was used to run all of the simulations in this study.

\section{REFERENCES}

Ansdell M. et al., 2016, ApJ, 828, 46

Ataiee S., Pinilla P., Zsom A., Dullemond C. P., Dominik C., Ghanbari J., 2013, A\&A, 553, L3

Bae J., Zhu Z., Hartmann L., 2016, ApJ, 819, 134

Barenfeld S. A., Carpenter J. M., Ricci L., Isella A., 2016, ApJ, 827, 142

Barge P., Sommeria J., 1995, A\&A, 295, L1

Barge P., Ricci L., Carilli C. L., Previn-Ratnasingam R., 2017, A\&A, 605 A122

Baruteau C., Zhu Z., 2016, MNRAS, 458, 3927

Bell C. P. M., Naylor T., Mayne N. J., Jeffries R. D., Littlefair S. P., 2013, MNRAS, 434, 806

Birnstiel T., Dullemond C. P., Brauer F., 2010, A\&A, 513, A79

Bohren C. F., Huffman D. R., 1983, Absorption and Scattering of Light by Small Particles. Wiley-Blackwell, New York

Bowler B. P., Nielsen E. L., 2018, preprint (arXiv:e-prints)

Casassus S. et al., 2013, Nature, 493, 191

Cazzoletti P. et al., 2018, preprint (arXiv:e-prints)

Clarke C. J., Pringle J. E., 1988, MNRAS, 235, 365

Crnkovic-Rubsamen I., Zhu Z., Stone J. M., 2015, MNRAS, 450, 4285

de Val-Borro M. et al., 2006, MNRAS, 370, 529

de Val-Borro M., Artymowicz P., D’Angelo G., Peplinski A., 2007, A\&A, 471,1043

Dipierro G., Laibe G., 2017, MNRAS, 469, 1932

Donati J. F. et al., 2016, Nature, 534, 662

Dong R., Zhu Z., Rafikov R. R., Stone J. M., 2015, ApJ, 809, L5

Dorschner J., Begemann B., Henning T., Jaeger C., Mutschke H., 1995, A\&A, 300, 503

Fu W., Li H., Lubow S., Li S., 2014, ApJ, 788, L41

Fu W., Li H., Lubow S., Li S., Liang E., 2014, ApJ, 795, L39

Fuente A. et al., 2017, ApJ, 846, L3

Garufi A. et al., 2013, A\&A, 560, A105

Hammer M., Kratter K. M., Lin M.-K., 2017, MNRAS, 466, 3533

Jaeger C., Mutschke H., Begemann B., Dorschner J., Henning T., 1994, A\&A, 292, 641

Johansen A., Andersen A. C., Brandenburg A., 2004, A\&A, 417, 361 
Keppler M. et al., 2018, A\&A, 617, A44

Kida S., 1981, J. Phys. Soc. Japan, 50, 3517

Kley W., 1999, MNRAS, 303, 696

Li H., Finn J. M., Lovelace R. V. E., Colgate S. A., 2000, ApJ, 533, 1023

Li H., Colgate S. A., Wendroff B., Liska R., 2001, ApJ, 551, 874

Li H., Li S., Koller J., Wendroff B. B., Liska R., Orban C. M., Liang E. P. T., Lin D. N. C., 2005, ApJ, 624, 1003

Lissauer J. J., Hubickyj O., D’Angelo G., Bodenheimer P., 2009, Icarus, 199,338

Lovelace R. V. E., Romanova M. M., 2014, Fluid Dyn. Res., 46, 041401

Lovelace R. V. E., Li H., Colgate S. A., Nelson A. F., 1999, ApJ, 513, 805

Lyra W., Lin M.-K., 2013, ApJ, 775, 17

Lyra W., Mac Low M.-M., 2012, ApJ, 756, 62

Masset F., 2000, A\&AS, 141, 165

Miranda R., Li H., Li S., Jin S., 2017, ApJ, 835, 118

Muto T. et al., 2012, ApJ, 748, L22

Owen J. E., Kollmeier J. A., 2017, MNRAS, 467, 3379

Pascucci I. et al., 2016, ApJ, 831, 125

Pérez L. M., Isella A., Carpenter J. M., Chandler C. J., 2014, ApJ, 783, L13

Pollack J. B., Hubickyj O., Bodenheimer P., Lissauer J. J., Podolak M., Greenzweig Y., 1996, Icarus, 124, 62

Price D. J. et al., 2018, MNRAS, 477, 1270

Ragusa E., Dipierro G., Lodato G., Laibe G., Price D. J., 2017, MNRAS, 464,1449

Regály Z., Juhász A., Sándor Z., Dullemond C. P., 2012, MNRAS, 419, 1701

Regály Z., Juhász A., Nehéz D., 2017, ApJ, 851, 89

Sallum S. et al., 2015, Nature, 527, 342
Shakura N. I., Sunyaev R. A., 1973, A\&A, 24, 337

Stolker T. et al., 2016, A\&A, 595, A113

Surville C., Mayer L., 2018, preprint (arXiv:e-prints)

Tanga P., Babiano A., Dubrulle B., Provenzale A., 1996, Icarus, 121, 158 van der Marel N. et al., 2013, Science, 340, 1199

van der Marel N., Pinilla P., Tobin J., van Kempen T., Andrews S., Ricci L., Birnstiel T., 2015, ApJ, 810, L7

van der Marel N., Cazzoletti P., Pinilla P., Garufi A., 2016, ApJ, 832, 178

Weidenschilling S. J., 1977, MNRAS, 180, 57

Youdin A. N., Lithwick Y., 2007, Icarus, 192, 588

Zhu Z., Nelson R. P., Dong R., Espaillat C., Hartmann L., 2012, ApJ, 755, 6

\section{SUPPORTING INFORMATION}

Supplementary data are available at MNRAS online. Movies corresponding to Figures 4 and 5 are provided.

Please note: Oxford University Press is not responsible for the content or functionality of any supporting materials supplied by the authors. Any queries (other than missing material) should be directed to the corresponding author for the article.

This paper has been typeset from a $\mathrm{T}_{\mathrm{E}} \mathrm{X} / \mathrm{L} \mathrm{AT} \mathrm{E}$ file prepared by the author. 